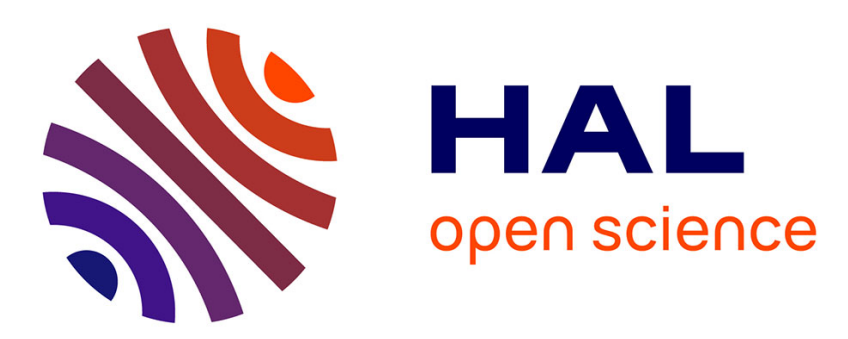

\title{
Avoir faim en Afrique occidentale française: investigations et représentations coloniales (1920-1960)
}

Vincent Bonnecase

\section{To cite this version:}

Vincent Bonnecase. Avoir faim en Afrique occidentale française: investigations et représentations coloniales (1920-1960). Revue d'histoire des sciences humaines, 2009, 21 (2), pp.151 - 151. 10.3917/rhsh.021.0151 . halshs-01618917

\section{HAL Id: halshs-01618917 https://shs.hal.science/halshs-01618917}

Submitted on 18 Oct 2017

HAL is a multi-disciplinary open access archive for the deposit and dissemination of scientific research documents, whether they are published or not. The documents may come from teaching and research institutions in France or abroad, or from public or private research centers.
L'archive ouverte pluridisciplinaire HAL, est destinée au dépôt et à la diffusion de documents scientifiques de niveau recherche, publiés ou non, émanant des établissements d'enseignement et de recherche français ou étrangers, des laboratoires publics ou privés. 


\title{
Avoir faim en Afrique occidentale française : investigations et représentations coloniales (1920-1960)
}

\author{
Vincent BONNECASE
}

\begin{abstract}
Résumé
Cet article retrace l'histoire de la faim, comme objet des savoirs coloniaux, en Afrique occidentale française des années 1920 aux années 1950. Dans l'entre deux-guerres, il y a un profond décalage entre la prétention coloniale à améliorer l'alimentation indigène et les connaissances effectives que les administrateurs ont de cette alimentation. Celleci est essentiellement appréhendée à partir d'indicateurs productifs. Il n'est alors que peu question d'évaluer les niveaux d'alimentation des populations colonisées à l'aune de critères nutritionnels. Au sortir de la Seconde Guerre mondiale, le contexte de crise des légitimités coloniales amène l'administration de l'AOF à produire un savoir nouveau sur les niveaux de vie des populations colonisées, notamment en termes d'alimentation. La faim n'en reste pas moins un phénomène mal connu dans la fédération jusqu'aux indépendances. Mais elle est devenue un objet d’investigations et de mesure.
\end{abstract}

Mots-clés : Faim - Famine - Construction des savoirs - Mesure des niveaux de vie Colonisation - Afrique occidentale française.

\begin{abstract}
Hunger in French Western Africa: Colonial Investigations and Representations (1920-1960)

This article focuses on the way Hunger was constructed as a specific colonial knowledge in French Western Africa from 1920 to 1960. During the Interwar period, there was a widening gap between the claim to improve the food standards of colonial subjects and the knowledge most administrators had on indigenous eating habits. Food intakes were measured through productive indicators rather than nutritional criteria. After World War Two, in the context of colonial legitimacies crisis, the colonial administration produced new knowledge on indigenous standards of living and more specifically on eating habits. There was no real improvement of the knowledge on Hunger before the independences but at least, this issue became an object of investigation and of measurement.
\end{abstract}

Key-words : Hunger - Famine - Building Knowledge - Living Standards Measurement - Colonization - French West Africa. 
La plupart des travaux relatifs à la question alimentaire en Afrique occidentale française se sont focalisés sur les famines bien plus que sur la faim ordinaire ${ }^{1}$. Cette focalisation historiographique reflète celle des sources, beaucoup plus disertes sur les grandes crises de subsistance que sur la sous-alimentation structurelle. Les sources orales donnent matière aux études de ces crises qui frappent partie ou totalité de l'Afrique occidentale française ${ }^{2}$, en particulier celles de 1913-1914, de 1931, de 1941-1942 ou de 1954 qui restent, dans les mémoires collectives des sociétés affectées, comme des marqueurs chronologiques ${ }^{3}$. Les sources administratives apportent quant à elles de nombreux éléments de connaissance sur certaines de ces famines - et notamment celle de 1931 qui, de ce fait, a été particulièrement étudiée ${ }^{4}$ tout en restant, par contraste, extrêmement discrètes sur la situation alimentaire hors période de crise.

On s'interrogera ici sur l'évolution des savoirs produits par l'administration coloniale sur la faim ordinaire en AOF des années 1920 aux années 1950, en mettant en question non seulement l'état de ces savoirs, mais aussi leur modalité concrète de construction sur le terrain. Au début des années 1920, les connaissances des administrateurs sur l'alimentation des populations colonisées découlent principalement des pratiques usuelles des membres des services coloniaux, en particulier ceux des services de l'Agriculture auxquels il appartient d'évaluer chaque année la production vivrière. Cette évaluation vise avant tout à prévenir le risque de famine. L'alimentation ordinaire des populations, quant à elle, n'est guère au centre des préoccupations coloniales. Quarante ans plus tard, le Gouvernement général dispose d'un petit nombre d'études spécifiquement consacrées à la question alimentaire. Celle-ci est appréhendée, non plus exclusivement à l'aune de la production, mais aussi sous l'angle nutritionnel. La sous-alimentation est ainsi devenue un objet d'investigations et de quantification. C'est cette histoire de la faim en AOF, comme objet des savoirs coloniaux, qu'il s'agit de retracer ici.

Trois traits essentiels nous semblent marquer ces savoirs. Le premier, le plus évident, tient à leur caractère globalement limité. L'ensemble des études réalisées sur l'alimentation des populations de l'AOF constitue un maigre corpus et, si l'on considère chacune de ces études, elles reposent elles-mêmes sur un matériau empirique extrêmement restreint. Ce premier trait tend à s'atténuer en fin de période coloniale, alors que se développe la recherche nutritionnelle : à partir de la fin des années 1940, une série d'enquêtes sur la ration alimentaire est menée dans des villes et des villages de la fédération, d'abord sans réel souci de représentativité, puis avec un véritable travail d'échantillonnage. Cela dit, le processus n'est qu'initié au terme des années 1950. Au moment des indépendances, alors que la lutte contre la faim a constitué, depuis le début de la conquête, un axe fort des discours de légitimation coloniale, les connaissances relatives au niveau d'alimentation des populations ouestafricaines restent sommaires.

\footnotetext{
${ }^{1}$ Hélène d'Almeida Topor consacre un article à la question de la faim en AOF, question qu'elle appréhende en croisant les données sur la production vivrière avec les données démographiques (ALMEIDA TOPOR, 1986).

${ }^{2}$ Désormais AOF.

${ }^{3}$ Il s'agit des principales crises énumérées par Boureima Alpha Gado pour l'Afrique sahélienne (ALPHA GADO, 1993).

${ }^{4}$ EGG, 1975 ; Fuglestad, 1974 ; SALIFou, 1975.
} 
Le deuxième trait caractéristique de ces savoirs tient à leurs mobiles, mobiles qui apparaissent largement extra-alimentaires. La période coloniale est ponctuée par des moments de brusques investigations sur l'alimentation des populations, au milieu des années 1920, à la fin des années 1930, ou dans la seconde partie des années 1940. Ces pics ne correspondent pas à des phases de brusque prise de conscience d'un problème alimentaire par les administrateurs : il n’y a pas de "découverte " à proprement parler. Elles correspondent bien plus à des phases de mise en question de la légitimité coloniale. L'immédiat après-guerre est à ce titre le moment le plus significatif. Au lendemain de la Seconde Guerre mondiale, les instances onusiennes tout juste créées exercent une nouvelle demande de savoirs sur les niveaux de vie des populations colonisées ${ }^{5}$. Dans un contexte de crise de la légitimité coloniale, les "puissances administrantes » sont amenées à produire du bilan et c'est dans ce cadre qu'il faut replacer la brusque phase d'investigations nutritionnelles de l'immédiat après-guerre.

Le dernier trait caractérisant les savoirs sur l'alimentation des populations de l'AOF tient à ce qu'ils restent étroitement circonscrits à un espace colonial. Dans les sociétés industrielles, à partir de la fin du XIX ${ }^{\mathrm{e}}$ siècle, des études sont menées pour mesurer, comparer, hiérarchiser les niveaux d'alimentation d'un pays à un autre ou d'une région à une autre ${ }^{6}$. Pareille optique n'est guère de mise en AOF durant la première moitié $\mathrm{du} \mathrm{xx}^{\mathrm{e}}$ siècle, les savoirs relatifs à l'alimentation des populations n'étant que peu régis par un souci de comparabilité. Cette absence de transversalité des savoirs se fonde partiellement sur un différentialisme racial, l'identité des besoins alimentaires entre Noirs et Blancs n'étant pas, alors, unanimement reconnue par les médecins et les nutritionnistes. Mais en deçà de cette caution scientifique, on pourra se demander si ce n'est pas d'abord l'entreprise coloniale de réification et d'essentialisation de l'altérité indigène qui est en jeu: jusqu'à la toute fin de la période coloniale, il ne semble guère question de mettre sur le même plan le niveau d'alimentation de populations colonisées et celui de populations qui ne le sont pas.

\section{I - Méconnaissances de l'entre-deux-guerres}

La prétention à maîtriser la situation alimentaire dans les colonies françaises est régulièrement réaffirmée dans l'entre-deux-guerres par les autorités ministérielles ou gouvernementales, délivrer les populations colonisées de la famine constituant une composante majeure de la mission civilisatrice ${ }^{7}$. En pratique, les connaissances des administrateurs sur le niveau d'alimentation des populations restent, en AOF, extrêmement limitées. Elles reposent essentiellement sur les indicateurs productifs établis dans le cadre de la gestion ordinaire des colonies. Quelques enquêtes nutritionnelles sont menées dans la fédération dans le cadre de vastes études de

\footnotetext{
${ }^{5}$ L'article 73 de la charte des Nations unies stipule ainsi que «les membres des Nations unies qui ont ou assument la responsabilité d'administrer des territoires dont les populations ne s'administrent pas encore complètement elles-mêmes (...) s'engagent (...) à communiquer régulièrement au secrétaire général des renseignements statistiques et autres de nature technique relatifs aux conditions économiques et sociales dans les territoires dont ils sont respectivement responsables » (Charte des Nations Unies, chapitre XI, article 73).

${ }^{6}$ Sur l'histoire des enquêtes alimentaires dans les sociétés industrielles, cf. BURNETT, 1995 pour l’Angleterre, CUlLATHER, 2007 pour les Etats-Unis, et LHUISSIER, 2007 pour la France.

${ }^{7}$ ConkLin, 1997.
} 
l'alimentation au sein de l'Empire, mais elles ne donnent lieu qu'à un savoir approximatif et non quantifiable de la sous-alimentation.

\section{L'alimentation sous l'angle de la production dans les années 1920}

Dans l'entre-deux-guerres, deux biais permettent à l'administration d'évaluer chaque année la production vivrière dans les colonies de l'AOF : une forme statistique avec le chiffrage de la production par les services agricoles et une forme visuelle avec la surveillance de greniers de réserves par les commandants de cercle. L'une et l'autre de ces deux formes manifestent un décalage permanent entre les prétentions coloniales et les réalités locales : elles témoignent d'une forte volonté de maîtrise de la situation alimentaire mais ne donnent que peu d'enseignements sur l'alimentation réelle des populations.

En 1915, un an après ce qui reste dans les mémoires collectives comme la plus grave famine du $\mathrm{xx}^{\mathrm{e}}$ siècle en Afrique de l'Ouest, François Clozel, gouverneur général de l'AOF, prescrit par circulaire «la constitution obligatoire de greniers de réserves ${ }^{8}$. L'année suivante, un arrêté général stipule que "tout indigène (...) se livrant à la culture des produits vivriers sera tenu de constituer à chaque récolte des approvisionnements de ses produits suffisants pour assurer sa nourriture, celle de sa famille ou celle de la collectivité pendant une année au moins ${ }^{9}$. Il s'agit, d'après les termes du gouverneur du Soudan relayant les nouvelles dispositions aux commandants de cercle, de « contraindre (l'indigène) à la prévoyance jusqu'à ce qu'il devienne lui-même prévoyant », car «s'il n’est pas exagéré d'affirmer que, dans de trop nombreuses régions encore, les populations n'ont pas pendant l'année entière une alimentation suffisante", c'est parce que "l'indigène est paresseux, apathique, il travaille le moins possible et il est d'une insouciance complète ${ }^{10}$. Durant tout l'entre-deux-guerres, cette thématique de l'imprévoyance indigène reste un leitmotiv dans les discours des administrateurs, dès lors qu'il y est question d'alimentation.

Le contrôle des réserves ainsi que la "prévoyance » que ce contrôle est supposé instaurer occupent ainsi une place importante dans l'activité du commandant de cercle, telle qu'elle peut être rétrospectivement décrite par d'anciens administrateurs ${ }^{11}$. L'idée de conserver des vivres jusqu'à la prochaine récolte n'est pourtant pas une invention coloniale: les nouveaux greniers ne font qu'institutionnaliser dans les collectivités villageoises des pratiques qui existent déjà, au moins à l'échelon familial ${ }^{12}$. Le changement réside dans l'encadrement administratif : dans chaque village, une quantité minimale de graines doit pouvoir être présentée par le chef de collectivité au commandant de cercle en tournée. En fait, cet

\footnotetext{
${ }^{8}$ Archives nationales du Mali, III/A825, Gouvernement général de l’AOF, circulaire aux gouverneurs, 24 juillet 1915.

9 Archives nationales du Mali, III/A825, Gouvernement général de l’AOF, arrêté du 13 septembre 1916

${ }^{10}$ Archives nationales du Mali, III/A825, Gouvernement du Soudan, circulaire aux commandants de cercle, 30 novembre 1916.

${ }^{11}$ Ainsi, pour Gabriel Massa, « chaque village devait construire des greniers, gérés par le chef de village et surveillés par l'administrateur. (...) Les greniers étaient mis à contribution en cas de disette ou simplement pour fournir la semence aux paysans imprévoyants » (MASSA, 2003, 170).

${ }^{12}$ AlPHA GADO, 1988, 2, 398.
} 
encadrement reste largement théorique, ne serait-ce que parce que le faible quadrillage du territoire par les administrateurs limite les risques de visite. Cette politique de réserves obligatoires se heurte en outre à une hostilité certaine des populations, ainsi que le relayent de nombreux administrateurs dès le début des années 1920 : prélever une partie de ses récoltes au bénéfice de l'administration ou des chefs de village, c'est comme se soumettre à une obligation fiscale ${ }^{13}$.

Parallèlement à l'institution de ces greniers, la volonté coloniale de maîtriser l'alimentation des populations colonisées se traduit également par l'apparition du chiffrage de la production vivrière au cours des années 1920. Au début de la décennie, les chefs des services agricoles se contentent, dans chaque colonie, d'évaluer brièvement dans leurs rapports annuels la récolte passée par un qualificatif tel que « médiocre », «bonne» ou « excellente ». Il arrive parfois que cette donnée soit accompagnée d'un tonnage approximatif de la principale production vivrière. Le gouverneur du Niger évalue ainsi en 1921 la récolte annuelle de mil à « 450 milliers de tonnes », tout en précisant que « les espaces propres à la culture du mil (...) sont à peu près infinis » et que "si le besoin s'en faisait sentir, on pourrait décupler l'importance de cette récolte ${ }^{14}$. L'idée, alors prédominante, de vastes espaces africains sous-peuplés va de pair avec celle de larges réserves de terres dont la colonisation agricole reste à faire.

C'est dans la seconde partie des années 1920 que les chiffres font leur apparition de manière systématique dans la nomenclature des rapports agricoles. À partir de 1926, les chefs de services doivent y faire apparaître les « statistiques de la production ou de l'exportation » pour les principales cultures. Ce changement de nomenclature n'est pas immédiatement suivi d'effets dans l'ensemble des colonies, certains chefs du service de l'Agriculture évoquant l'impossibilité, «à cause de l'état actuel de (leurs) moyens d'investigation, de déterminer la superficie exacte des terrains de culture indigène ${ }^{15}$, d'autres se contentant de transmettre les chiffres des exportations notifiés par le service des Douanes ${ }^{16}$ ou d'établir un "aperçu de la production dans certains cercles $»{ }^{17}$. Tous finissent néanmoins par s'y soumettre à la fin de la décennie, l'ensemble des chefs des services agricoles de la fédération étant alors à même de proposer un tonnage pour les principales cultures vivrières de leur colonie.

Cette évolution est couronnée, en 1930, par la création des «Offices de l'alimentation indigène » dans chaque colonie de l'AOF. Dirigés par le secrétaire général de la colonie, ils ont également pour membres le président de la Chambre de

\footnotetext{
${ }^{13}$ Ainsi que le reconnaît Henri Labouret, ancien administrateur devenu professeur à l’École nationale de la France d'outre-mer, « cette institution [des greniers de réserves], qui a été considérée comme une panacée contre la famine et la disette, se fondait en réalité sur l'ignorance des vraies conditions locales chez ceux qui la préconisaient (...) : en admettant qu'une telle organisation soit possible, il serait très difficile de la rendre efficace, les propriétaires de grains se refusant à distraire une partie de leur récolte pour la placer dans le silo ou le grenier du village ou du canton administré par le chef (LABOURET, 1938, 440-441).

14 Archives nationales du Sénégal, 2G21.24, colonie du Niger, rapport agricole annuel, 1921. Le gouverneur envisage dans le même rapport une production intensive d'alcool de mil comme « combustible nécessaire à l'industrialisation de demain ».

${ }^{15}$ Archives nationales du Sénégal, 2G26.91, colonie du Niger, rapport économique annuel, 1926.

${ }^{16}$ Au Soudan par exemple, seuls sont transcrits les chiffres de la production exportée, chiffres qui font apparaître une sortie de produits vivriers mais dans de faibles quantités (Archives nationales du Sénégal, 2G26.31, colonie du Soudan, rapport agricole annuel, 1926).

${ }^{17}$ Archives nationales du Sénégal, 2G26.31, colonie de Haute-Volta, rapport agricole annuel, 1926.
} 
commerce, le chef du service de l'Agriculture et deux notables indigènes. Ils ont pour fonction d'établir chaque année un bilan général des disponibilités alimentaires en rapportant, pour chaque cercle, les chiffres de la production vivrière et ceux de sa population ${ }^{18}$. Ces derniers reposent sur les recensements administratifs périodiquement organisés dans les circonscriptions par les commandants de cercle en tournée ${ }^{19}$. Ce bilan général doit permettre d’organiser le transfert de denrées depuis les cercles excédentaires jusqu'à ceux dont la production est jugée insuffisante pour permettre le ravitaillement de leur population.

Cette politique du chiffrage traduit surtout une évolution dans la mise en forme de l'information vivrière : concrètement, les modes d'évaluation de la production, eux, n'évoluent pas. Ils reposent sur une évaluation spatiale des cultures par les administrateurs, chacun d'eux estimant le nombre d'hectares affectés à chaque culture dans son cercle avant de transmettre les données au chef du service de l'Agriculture. En pratique, chaque administrateur a tendance à reproduire les mêmes chiffres d'année en année, de telle sorte qu'une brusque variation statistique à l'échelle d'un cercle traduit le plus souvent, non pas une brusque évolution de la production, mais un changement d'administrateur. Une fois les chiffres de la superficie centralisés par le chef du service de l'Agriculture, celui-ci applique, pour chaque culture, un rendement unique à l'échelle de la colonie ${ }^{20}$. Les chiffres de la population sur lesquels repose l'évaluation des besoins sont, quant à eux, tout aussi approximatifs, les recensements administratifs à finalité fiscale aboutissant systématiquement à une sous-évaluation des effectifs ${ }^{21}$.

L'incapacité administrative à prévoir les crises alimentaires est le meilleur signe de cette hasardeuse production statistique. En 1931, a lieu au Niger la principale des famines qui affecte l'AOF dans l'entre-deux-guerres. Le $1^{\mathrm{er}}$ juillet, une « Commission spéciale » est constituée dans la colonie après que « la rumeur publique a apporté des bruits de famine ${ }^{22}$. Présidée par le gouverneur, Louis Blacher, elle a pour membres le secrétaire général de la colonie, les chefs des services de l'Agriculture, de l'Administration générale et des Affaires économiques, ainsi que le commandant de cercle de Niamey et son adjoint. Le procès-verbal de la première séance traduit, chez ces plus hauts responsables administratifs de la colonie, une appréciation totalement empirique de la crise. Le commandant de cercle de Niamey et son adjoint signalent que des "faméliques" sont apparus depuis le mois de mai dans la ville. Les mendiants, « vieillards pour la plupart », y semblent plus nombreux que d'habitude. Dans les campagnes, certaines populations sont dans un « état squelettique » et des

\footnotetext{
${ }^{18}$ Archives nationales du Mali, III/1Q2879, Gouvernement général de l’AOF, arrêté du 22 mars 1930

${ }^{19}$ En vertu d'une instruction ministérielle datant du 15 février 1909, les chiffres de la population doivent être actualisés chaque année au moyen de recensements collectifs organisés dans les villages et les centres urbains par les administrateurs (Archives nationales du Burkina Faso, 8V315, ministère des Colonies, «Instruction ministérielle relative à l'établissement des statistiques dans les colonies », 15 février 1909).

${ }^{20}$ Archives nationales du Sénégal, 2G32.64, colonie du Niger, rapport agricole annuel, 1932.

${ }^{21}$ Les premières enquêtes démographiques par sondage réalisées en Afrique occidentale à la fin des années 1950 et au début des années 1960 concluent à une sous-estimation des effectifs par les recensements administratifs de l'ordre de 20 \% (DAKUYO, LOUGUÉ, 1984, 2, 170).

${ }^{22}$ Archives nationales du Sénégal, 11G26, colonie du Niger, « Procès-verbal de la commission réunie le $1^{\mathrm{er}}$ juillet 1931 pour examiner les possibilités et les modes de ravitaillement de la population indigène atteinte par la crise alimentaire », $1^{\mathrm{er}}$ juillet 1931.
} 
files de personnes se constituent aux abords des postes administratifs pour y recevoir des secours. Le secrétaire général fait quant à lui état de "femmes rencontrées au cours de ses promenades (...) accompagnées d'enfants paraissant sous-alimentés ». Il cite le cas d'un "enfant de trois ans qu'il a trouvé, dans les rochers, près de la résidence ${ }^{23}$.

En dehors de ces signes visibles, nul indicateur ne permet d'évaluer l'ampleur de la crise. Le gouverneur demande plusieurs fois au cours de la réunion des données chiffrées sur l'augmentation de la mortalité dans le cercle de Niamey, sans obtenir de réponse ${ }^{24}$. Quant aux statistiques vivrières de la précédente récolte, ils laissaient prévoir une "possibilité de soudure", d'après les termes du chef des Affaires économiques qui s'étonne que le commandant de cercle de Niamey ait dû procéder à des distributions gratuites de vivres ${ }^{25}$. Ce n'est finalement que tardivement - et rétrospectivement - que le service de l'Agriculture reconnaît l'existence d'une sousproduction vivrière. Dans le rapport annuel de 1930, rédigé après la récolte, celle-ci avait été jugée inférieure à la normale sans que soit mise en cause la possibilité de la colonie de subvenir à ses propres besoins ${ }^{26}$. Dans le rapport de 1931, il est question d'une « disette » dans les cercles de l'ouest de la colonie ${ }^{27}$. Dans celui de 1932, il est question de « la terrible famine de 1931 ». Le chef du service de l'Agriculture admet alors que les précédentes récoltes avaient été largement surestimées, dès lors que les quantités relevées auraient dû être «suffisantes pour assurer l'alimentation normale des Nigériens ${ }^{28}$.

Si le chiffrage de la production vivrière s'avère comme un piètre moyen de contrôler la situation alimentaire, on peut en dire autant de la politique des réserves obligatoires, laquelle ne permet pas à l'administration d'anticiper sur la famine de 1931. En 1932, le gouverneur général de l’AOF, Jules Brévié, reconnaît dans une circulaire adressée aux gouverneurs que « la constitution de réserves de vivres ne peut être réglementée d'une façon aussi stricte » et admet jusqu'à «la réticence des indigènes à parler de leur récolte » devant des représentants de l'administration. Il finit par appeler les administrateurs à « faciliter l'installation de greniers de réserves de vivres en employant tous les moyens de persuasion ${ }^{29}$. Le gouverneur général reconnaît par ces termes le caractère non contraignant des dispositions administratives prises jusqu'alors en faveur des greniers de réserves et le fait que ceux-ci ne permettent en aucun cas aux administrateurs de prendre la mesure de la situation vivrière.

\footnotetext{
${ }^{23}$ Ibid.

${ }^{24}$ Le gouverneur semble à cette occasion découvrir la faible emprise qu'ont les administrateurs sur la réalité sanitaire et démographique de la colonie, d’après ses propos, adressés au commandant de cercle de Niamey: «il faut préciser, donnez-moi un chiffre. Vous êtes cinq Européens au cercle, vous êtes commandant de cercle, vous devez pouvoir m'indiquer si dans telle ou telle région, il meurt 10, 20, 30 ou 40 personnes. Lorsqu'une épidémie sévit dans la colonie, nous sommes bien informés du nombre de décès ? » (ibid.).

${ }^{25}$ Ibid.

${ }^{26}$ Ce jugement est réitéré dans deux rapports de l'Office de l'alimentation du Niger établis en janvier et en mars 1931 (Archives nationales du Sénégal, 11G26, lettre du gouverneur du Niger au gouverneur général de l’AOF, 13 novembre 1931).

${ }^{27}$ Archives nationales du Sénégal, 2G31.60, colonie du Niger, rapport agricole annuel, 1931.

${ }^{28}$ Archives nationales du Sénégal, 2G32.64, colonie du Niger, rapport agricole annuel, 1932.

${ }^{29}$ Archives nationales du Mali, III/1Q2879, Gouvernement général, circulaire aux gouverneurs, 13 septembre 1932.
} 
Ainsi, le suivi de la production vivrière en $\mathrm{AOF}$, que ce soit par le biais des statistiques agricoles ou des réserves obligatoires, ne donne aux administrateurs qu'une connaissance lacunaire du niveau d'alimentation des populations dans leurs cercles ou leurs colonies au cours des années 1920, ceci apparaissant clairement - et rétrospectivement - à la lumière de la crise de 1931.

\section{Les premières enquêtes alimentaires des années 1930}

Dans les années 1930, si le savoir colonial relatif à l'alimentation continue de reposer essentiellement sur le critère productif, des premières recherches nutritionnelles sont initiées en AOF. Elles consistent en des études générales fondées sur les observations des administrateurs et en quelques enquêtes plus précises menées sur le régime alimentaire de populations dans des cercles de la fédération. Ce savoir, extrêmement empirique, débouche sur un constat global de sous-alimentation, sans toutefois en évaluer l'ampleur.

«L'Étude de la ration alimentaire des indigènes du territoire du Togo », faite en 1925 par le pharmacien-colonel Cheyssial, est la première à chiffrer la ration calorique moyenne dans une colonie française. Elle crée en cela un précédent auquel se réfèrent les études ultérieures menées en $\mathrm{AOF}^{30}$. Elle est suscitée par les propos tenus la même année à l'Académie des Sciences coloniales par le professeur Roubaud selon lequel « les populations indigènes ne trouvent que rarement, dans les ressources vivrières auxquelles elles font appel, les conditions d'une ration normale d'entretien ${ }^{31}$. En réaction, le ministre des Colonies, Edouard Daladier, demande dans une instruction adressée à l'ensemble des gouverneurs de procéder au calcul de «la ration indigène " ${ }^{32}$. Cette demande est suivie d'effet dans le seul Togo, territoire sous mandat de la Société des Nations, déjà soumis en cela à un contrôle particulier ${ }^{33}$. L'étude de 1925 délivre des chiffres sur la ration annuelle moyenne d'individus issus des six principales ethnies de la colonie. Il ressort de ces chiffres que « les indigènes du Togo (...), loin d'être sous-alimentés, sont au contraire sur-alimentés » : hormis un cas, les moyennes calculées sont toutes supérieures à 3500 calories et s'élèvent jusqu'à 6500 calories par jour et par personne ${ }^{34}$. L'objectif initial, démentir les allégations de sous-alimentation dans l’Empire, est en cela rempli.

${ }^{30} 12$ ans après, ces recherches sur l'alimentation sont encore citées par Henri Labouret, professeur à l’École nationale de la France d’outre-mer, comme «les seules qui aient été poursuivies (...) de façon méthodique et scientifique dans un territoire placé sous l'administration française » (LABOURET, 1938, 438).

${ }^{31}$ RoUBAUD, 1926, 357-376.

${ }^{32}$ Ministère des Colonies, « Instruction aux gouverneurs généraux relative à l'étude hygiénique de la ration alimentaire des populations indigènes ", 1925, in HARDY, RICHET, 1933, annexe.

${ }^{33}$ Les territoires sous mandat - anciennes possessions allemandes ou ottomanes placées par la Société des Nations sous la tutelle d'un Etat européen après la Première Guerre mondiale - devaient donner lieu, de la part de la puissance mandatée, à la rédaction d'un rapport annuel à la SDN d'après l'article 23 de son pacte.

${ }^{34}$ HARDY, RICHET, 1933, 278-289. Cinq des sept rations calculées sont comprises entre 4800 et 6500 calories, les deux autres s’élevant à 3553 et 2529 calories. Selon les termes d’A. Cheyssial, « chez une seule race, celle des Lossos (trop paresseuse pour travailler), on observe une carence alimentaire que les intéressés pourraient facilement faire disparaître par un travail plus sérieux ». 
Quelques années plus tard, Georges Hardy et Charles Richet, respectivement directeur de l'Ecole nationale de la France d'outre-mer et professeur spécialiste des problèmes alimentaires à la Faculté de médecine de Paris, entreprennent de synthétiser les savoirs des administrateurs et des médecins coloniaux sur "l'alimentation indigène dans les colonies françaises » ${ }^{35}$. Le produit de leurs investigations est publié en 1933. Dans l'introduction, Georges Hardy et Charles Richet se placent d'emblée dans une perspective de valorisation de la mission civilisatrice, affirmant que «chaque fois qu'un peuple européen a conquis un pays colonial, il s'est trouvé en face de régions dont la plupart des habitants avaient une nourriture insuffisante " et que "dès que les militaires, les administrateurs, les missionnaires, les ingénieurs, les médecins, c'est-à-dire la civilisation blanche, a pris possession d'un pays, la famine a disparu ». Ils estiment en même temps «qu'il y a encore $30 \%$ des indigènes qui s'alimentent insuffisamment dans (les) territoires d'outre-mer ${ }^{36}$.

L'ouvrage de Georges Hardy et de Charles Richet traduit surtout le peu de données proprement nutritionnelles dont disposent les administrateurs et les médecins coloniaux pour juger de la situation alimentaire sur leurs territoires. La hiérarchisation des niveaux d'alimentation dans les différentes fédérations et colonies de l'Empire repose ainsi sur un étalage sommaire des différentes maladies de carence alimentaire qui y ont été constatées par les services de Santé. Sur ce critère, l'alimentation en AOF est jugée « insuffisante dans certains districts », ce qui est mieux qu'en Afrique équatoriale française où elle est "très insuffisante " au regard des nombreux cas « d'avitaminose $\mathrm{E}$ » et de «misère physiologique » relevés par les administrateurs. C'est également mieux que dans d'autres parties de l'Empire telles que l'Indochine ou Madagascar, associées au « béribéri » ${ }^{37}$.

Finalement, transparaît de cette compilation que l'AOF est affectée par une sousalimentation ordinaire, même si elle apparaît mieux lotie que d'autres parties de l'Empire. Cette appréciation, aussi minimale soit-elle, n'apparaît pas comme une évidence communément partagée au début des années 1930. Henri Labouret, ancien officier des troupes coloniales devenu administrateur puis professeur à l'Ecole nationale de la France d'outre-mer est auteur, dans l'ouvrage de Georges Hardy et de Charles Richet, d'une contribution sur «l'alimentation des indigènes en AOF » ${ }^{38}$. Il y fustige l'image d'une "Afrique (...) considérée par le public comme l'une des régions les plus fertiles du monde dans laquelle vivent des indigènes paresseux que nourrit sans peine une nature généreuse ». Contre cette image, il affirme que " presque partout le nègre est sous-alimenté », sans toutefois pouvoir étayer cette assertion du moindre chiffre ${ }^{39}$.

35 Georges Hardy, ancien professeur devenu inspecteur général puis directeur général de l'Enseignement en AOF, dirige l'École nationale de la France d'outre-mer de 1926 à 1932. Quant à Charles Richet, il s’agit du fils de Charles Richet, prix Nobel de médecine en 1913.

${ }^{36}$ HARDY, RICHET, 1933, 289.

${ }^{37}$ Ibid., 40.

${ }^{38}$ Henri Labouret est officier des troupes en coloniales en Côte d'Ivoire jusqu'en 1912, avant d'exercer les fonctions d'administrateur au Haut-Sénégal-Niger, puis en Haute-Volta. En 1926, il succède à Henri Delafosse comme responsable des cours portant sur l'Afrique occidentale française à l'École nationale de la France d'outre-mer.

${ }^{39}$ HARDY, RICHET, 1933, 152. 
Ce n'est que six ans plus tard que sont initiées les premières études de la ration alimentaire en AOF. Elles s'inscrivent dans le cadre des travaux de la commission parlementaire instituée en janvier 1937 par le gouvernement du Front populaire pour enquêter sur « les besoins et les aspirations légitimes des populations habitant les colonies » ${ }^{40}$. La Commission d'enquête, qui se réunit pour la première fois en juillet 1937, comprend plusieurs sous-commissions géographiques, celle consacrée à l'Afrique étant présidée par Henri Labouret ${ }^{41}$. L'un des principaux travaux des commissionnaires consiste, dans un premier temps, à établir un canevas d'enquête commun à l'Empire pour jeter les bases d'un savoir comparatiste sur les niveaux de vie dans les colonies. Plusieurs domaines sont prospectés parmi lesquels le domaine alimentaire. En février 1938, un questionnaire sur l'alimentation est ainsi envoyé par la Commission d'enquête dans l'ensemble des colonies françaises ${ }^{42}$. Le ministre des Colonies, Marius Moutet, demande par circulaire adressée aux gouverneurs que le questionnaire soit diffusé dans chaque poste administratif et, cela, en nombre suffisant pour que, «s'il existe dans un même poste des races de mœurs différentes, (...) le médecin en dispose d'un pour chaque race $"{ }^{43}$.

Ce questionnaire invite les administrateurs à créer un savoir nutritionnel véritablement nouveau sur les colonies. Outre l'information habituelle sur la production vivrière dans chaque cercle, les chefs de circonscription doivent fournir une étude détaillée de la ration alimentaire de quatre ou cinq familles parmi lesquelles au moins une «famille riche », une « famille aisée » et une " famille pauvre ». Cette étude est confiée à des fonctionnaires africains, généralement des instituteurs, chargés d'assister aux repas familiaux et de peser les différents aliments entrant dans leur composition. Elle doit durer « au moins six mois » de telle sorte à prendre en compte les variations de régime selon la saison. Les différences de régime entre les membres d'une même famille, selon le sexe et selon l'âge, doivent également être notifiées ${ }^{44}$. C'est la première fois qu'un tel programme de recherches nutritionnelles, avec des critères d'évaluation standardisés, est mené dans l'Empire français. La considération d'une stratification sociale, interfamiliale et intrafamiliale, est en outre assez remarquable en ce qu'elle rompt avec l'image de sociétés africaines dont la supposée solidarité traditionnelle aplanirait les disparités.

Ce programme n'est pas mené à terme. La chute du Front populaire en avril 1938 et la dissolution de la Commission d'enquête en juillet 1938 interviennent avant que

${ }^{40}$ Ce sont les termes de la loi du 30 janvier 1937 qui institue la Commission d'enquête sur les territoires d'outre-mer. Celle-ci est composée, lors de sa première séance, de 42 membres dont 15 parlementaires, sept anciens administrateurs coloniaux, deux missionnaires, deux syndicalistes, deux fonctionnaires internationaux, deux avocats et 12 intellectuels parmi lesquels André Gide et Lucien LévyBruhl (Archives nationales du Sénégal, 17G252, ministère des Colonies, décrets du 4 février 1937 et du 19 mai 1937 relatifs à la composition et au fonctionnement de la Commission d'enquête sur les territoires de la France d'outre-mer).

${ }^{41}$ Archives nationales du Sénégal, 17G252, Commission d'enquête sur les territoires de la France d’outre-mer, procès-verbal de la séance inaugurale tenue au ministère des Colonies, 8 juillet 1931.

${ }^{42}$ Deux autres questionnaires sont envoyés au même moment à l'ensemble des gouverneurs, l'un sur l'habitation et les conditions sanitaires des indigènes et, l'autre, sur les métis. Un quatrième sur l’immigration est envoyé au seul gouverneur de l'Indochine.

43 Archives nationales du Sénégal, 17G252, ministère des Colonies, circulaire aux gouverneurs généraux et aux gouverneurs des colonies, 14 février 1938.

${ }^{44}$ Archives nationales du Mali, I/5D1938, Commission d'enquête sur la France d'outre-mer, « Enquête n 1C sur l'alimentation indigène », 1938. 
les résultats ne soient centralisés et exploités, le travail n'ayant pas même été commencé dans bon nombre de circonscriptions ${ }^{45}$. La fin du Front populaire n'entraîne toutefois pas la cessation immédiate des investigations. Le successeur de Marius Moutet au ministère des Colonies, Georges Mandel, demande dans une circulaire envoyée en mai 1939 aux gouverneurs que lui soient retournées les réponses au questionnaire sur l'alimentation ${ }^{46}$. À l'échelle de l'AOF, les gouverneurs sont en mesure de fournir ces réponses pour une minorité de circonscriptions : celui du Soudan peut se prévaloir du plus grand nombre de résultats avec 19 réponses, suivi par les gouverneurs de la Côte d'Ivoire, du Sénégal et du Niger avec, respectivement, onze, 6 et 5 réponses ${ }^{47}$. Cette prééminence du Soudan peut s'expliquer par le rôle alimentaire particulier prêté à la colonie dans les années 1930, les grands travaux hydrauliques et culturaux réalisés à partir de 1932 par l'Office du Niger dans la zone de Ségou visant à produire du riz pour l'ensemble de l'AOF ${ }^{48}$.

Le contenu desdites réponses reste en deçà des attentes de départs formulées par les membres de la Commission d'enquête. Rares sont celles à prendre en compte des différences de régime entre "familles pauvres » et «familles riches » et, plus rares encore, celles à notifier des disparités intrafamiliales ${ }^{49}$. Celles qui le font mettent toutefois à mal le stéréotype d'une solidarité traditionnelle propre à "l'Afrique " encore largement véhiculé par bon nombre d'administrateurs ${ }^{50}$. L'un des principaux résultats communément admis consiste à reconnaître les variations saisonnières de régimes. S'il ne s'agit pas d'une découverte, pareille variation n'a jusqu'alors jamais donné lieu à des études sur des familles suivies pendant une période de plusieurs mois. Outre l'amenuisement de la ration en période de soudure, cela amène les administrateurs à lister, dans les cercles étudiés, les aliments de substitution qui entrent dans sa composition. Finalement, l'enquête du Front populaire pose les bases d'un savoir nutritionnel nouveau en AOF sans aller au-delà de ces bases.

À la fin des années 1930, les savoirs coloniaux sur l'alimentation des populations de l’AOF restent donc insignifiants. Reste à nuancer la spécificité coloniale de cet état

\footnotetext{
${ }^{45}$ Les membres de la Commission d'enquête démissionnent collectivement le 8 juillet 1938 (AGERON, COQUERY-VIDROVITCH, MEYNIER, THOBIE, 1990, tome 2, 260).

46 Archives nationales du Sénégal, 17G252, ministère des Colonies, circulaire aux gouverneurs généraux et aux gouverneurs des colonies, 3 mai 1939.

${ }^{47}$ Archives nationales du Sénégal, 17G252, lettre du gouverneur du Soudan au gouverneur général de l'AOF, 30/3/1939; lettre du gouverneur de la Côte d'Ivoire au gouverneur général de l'AOF, 11 juin 1939 ; lettre du gouverneur du Sénégal au gouverneur général de l’AOF, 9 juin 1939 ; lettre du gouverneur du Niger au gouverneur général de l’AOF, 24 juillet 1939.

${ }^{48}$ Pour une histoire récente de l'Office du Niger, $c f$. BEUSEKOM, 2000. Cf. aussi la thèse de Jean FILIPOVICH (1985).

${ }^{49}$ Dans le cercle de Kita, l'enquêteur distingue le grand champ où tous cultivent le matin et dont les produits sont affectés aux dépenses communes, les champs du ménage où travaille l'après-midi chacun des frères du chef de famille avec ses femmes et ses enfants et les champs du soir où peuvent travailler individuellement chaque fils majeur et chaque femme « afin d'améliorer sa propre alimentation ou celle de ses enfants ». Des degrés de ressources peuvent donc se dessiner entre les membres d'une même famille élargie, les champs de l'après-midi et du soir étant, excepté en période de disette, « destinés à améliorer l'alimentation du groupe restreint » (Archives nationales du Mali, I/5D1938, cercle de Kita, rapport d'enquête $n^{\circ} 1 \mathrm{C}$ sur l'alimentation des indigènes, non daté).

${ }^{50}$ Ainsi le commandant de cercle de Kayes présente-t-il un budget type d'une famille qui est «pour ainsi dire le même chez tous les noirs agriculteurs » (Archives nationales du Mali, I/5D1938, cercle de Kayes, cantons du Khasso, du Dioboko et du Logo, rapport d'enquête $n^{\circ} 1 \mathrm{C}$ sur l'alimentation des indigènes, non daté).
} 
de fait. Si la recherche nutritionnelle se développe véritablement en Europe occidentale à partir de la fin du $\mathrm{XIX}^{\mathrm{e}}$ siècle, la France reste à la traîne en la matière par rapport à des pays comme l'Angleterre ou l'Allemagne ${ }^{51}$. Ce n'est qu'en 1937 qu'est créé en métropole un service d'enquête nationale sur l'alimentation et les premières données collectées ne sont pas exploitées avant la Seconde Guerre mondiale ${ }^{52}$. Il ne s'agit pas de nier l'indigence des recherches nutritionnelles menées en AOF durant l'entre-deux-guerres mais juste de la relativiser à l'aune de la temporalité scientifique métropolitaine.

\section{II - La construction d'un savoir nutritionnel dans l'après-guerre}

L'immédiat après-guerre voit s'éclore une demande internationale de savoir sur les colonies. Dans un contexte de forte mise en cause de la colonisation au sein des nouvelles organisations onusiennes, les puissances coloniales sont appelées à produire une information nouvelle sur les conditions de vie des populations colonisées. Cette demande de savoir s'exerce notamment sur la question de l'alimentation. Dès mai 1943, à la conférence de Hot Springs qui pose les bases de l'Organisation des Nations unies pour l'Alimentation et l'Agriculture, les États participants s'engagent à envoyer à la future organisation des rapports périodiques sur la situation alimentaire dans leur territoire et « ceux qu'ils ont à charge », rapports qui doivent être " comparables d'un pays à l'autre, (...) conçus sur un plan rigoureusement identique et établis selon les mêmes méthodes, pour donner des conclusions du même ordre $"{ }^{53}$. Cette production d'un savoir standardisé sur l'alimentation dans les colonies françaises ne va pas de soi dans l'après-guerre, tant le présupposé de standings propres aux populations colonisées est vivace.

\section{La raciologie de la mission anthropologique à la fin des années 1940}

En mai 1946, le gouverneur général de l'AOF, René Barthès, crée par arrêté «l'Organisme d'enquête pour l'étude anthropologique des populations indigènes de l'AOF (alimentation et nutrition) », communément désigné sous le nom de « Mission anthropologique ${ }^{54}$. Le savoir produit par cet organisme dans la seconde partie des années 1940 apparaît profondément ambivalent. D’un côté, le travail de la Mission constitue l'une des premières entreprises de quantification de la ration alimentaire dans les colonies françaises d'Afrique, établie à des fins de comparaison internationale et au moyen de procédés d'enquête bien explicités. De l'autre, ses orientations de recherches sont largement empreintes des postulats de l'anthropologie coloniale de l'entre-deux-guerres avec, en particulier, le paradigme racial qui reste

\footnotetext{
${ }^{51}$ SIMMONS, 2004.

${ }^{52}$ DUPIN, 1969, 10

${ }^{53}$ Centre des Archives d'outre-mer, 1affpol356, Organisation des Nations unies pour l'Alimentation et l’Agriculture, rapport préliminaire à la première session, « Projet de canevas unique pour l'établissement du rapport annuel spécialement dans les pays à développement retardé », 1945.

${ }^{54}$ Fonds de l'Institut de Médecine tropicale du service de Santé des armées, 101, rapport de Léon Pales sur l'Organisme d'enquête pour l'étude anthropologique des populations indigènes de l'AOF (alimentation et nutrition), 10 octobre 1945.
} 
posé comme une clef d'explication possible des profils et des comportements alimentaires.

Si l'on considère d'abord les conditions concrètes dans lesquelles est produit ce savoir, l'une de ses caractéristiques réside dans le peu d'individus sur lequel il repose, qu'il s’agisse des enquêteurs ou des personnes étudiées. À sa création, la Mission anthropologique comprend cinq médecins et quatre pharmaciens, tous issus des troupes coloniales. Elle bénéficie en outre du soutien d'auxiliaires techniques, essentiellement des chauffeurs et des interprètes, ainsi que de celui de chimistes basés à Dakar. Le chef de cette équipe, Léon Pales, a été médecin au Congo et au Tchad dans les années 1930 avant de devenir, en 1938, professeur d'anthropologie à l'École de médecine des troupes coloniales à Marseille, en 1943, sous-directeur du Musée de l'Homme à Paris et, en 1945, adjoint technique à la Direction générale de la Santé publique de l'AOF. Sur le plan de la recherche, il est notamment connu pour avoir mené en 1938 des investigations sur un bataillon de tirailleurs basé à Marseille afin, d'après ses propres termes, «d'améliorer la connaissance anthropologique des Soudanais occidentaux sélectionnés et transplantés en métropole $»{ }^{55}$.

Les premières recherches de terrain sont menées entre 1946 et 1948 au Sénégal, au Soudan, en Guinée et au Soudan. Quatre tournées successives sont organisées par Léon Pales, chaque équipe comprenant un maximum de trois chercheurs, membres de la Mission anthropologique, et de cinq auxiliaires techniques. Au total, 20000 kilomètres de route sont parcourus sur une période cumulée de quinze mois et, cela, jamais par plus de huit personnes à la fois ${ }^{56}$. Le nombre de personnes observées dans le cadre des enquêtes alimentaires est à la mesure de celui des enquêteurs. 500 enquêtes familiales sont menées dans les cinq colonies parcourues avec, pour objectif, le calcul de la valeur qualitative et quantitative de la ration individuelle moyenne. Une enquête consiste à passer huit jours dans une famille, de peser les aliments consommés, de noter leur mode de préparation et d'assister au repas. Elle porte donc sur les seuls apports visibles, c'est-à-dire sur « ceux qui figurent au menu familial et qui font l'objet d'une préparation, les seuls dont fasse état l'Africain », d'après les termes de Léon Pales ${ }^{57}$. Elle occulte totalement les compléments relevant de la chasse ou de la cueillette. Certains aliments consommés n’apparaissent ainsi presque jamais, en particulier les fruits qui, dans aucune des enquêtes, ne sont mentionnés ${ }^{58}$.

Outre ces recherches relatives à la ration, des enquêtes biochimiques sont également faites à Dakar afin de mesurer les éventuelles carences nutritionnelles. Elles consistent à effectuer des prélèvements sanguins sur des populations, comprenant généralement une bonne proportion de militaires, pour en faire l'analyse. Les premières recherches biochimiques sur les avitaminoses sont ainsi faites en 1948

\footnotetext{
${ }^{55}$ BONNECASE, 2008, 167.

${ }^{56}$ Archives de l'Office du Niger, S0166, Organisme d'enquête pour l'étude anthropologique des populations indigènes de l'AOF, «Le bilan de la mission anthropologique de l'AOF (janvier 1946-août 1948) », 1948.

${ }^{57}$ Ibid. Parmi ces 500 enquêtes familiales, 260 sont menées au Soudan, 98 au Sénégal, 72 en Guinée et 70 en Haute-Volta.

${ }^{58}$ Fonds de l’Institut de Médecine tropicale du service de Santé des armées, 101, Organisme de Recherches sur l'Alimentation et la Nutrition africaines, «L'alimentation en AOF. Milieux, enquêtes, techniques, rations », 1954.
} 
sur un échantillon de 250 personnes, dont un tiers de tirailleurs ${ }^{59}$. La question de la représentativité des échantillons observés n’est jamais abordée par les membres de la Mission anthropologique sauf, précisément, dans le cas spécifique des tirailleurs. Face à la critique selon laquelle ses résultats se fonderaient sur une « élite physique qui ne traduirait pas le faciès réel des populations dont elle est issue ", Léon Pales met en exergue la «tendance des meilleurs éléments d'une collectivité à se soustraire au service » : le choix d'un échantillon de militaires, plus commode à étudier, peut donc aussi bien jouer dans le sens d'une sur-évaluation que d'une sous-évaluation de la population étudiée ${ }^{60}$.

Ces conditions de production du savoir par la Mission anthropologique peuvent paraître paradoxales quand on pense qu'il est destiné à une diffusion internationale. Il peut par exemple paraître étrange que le premier rapport sur « les avitaminoses en AOF », transmis en 1948 aux Nations Unies, repose sur un matériau brut de 250 prélèvements sanguins. Ceci s'inscrit cependant dans un contexte international d'ignorance partagée sur l'alimentation en Afrique. En octobre 1949, a lieu à Dschang, au Cameroun, la première " conférence inter-africaine sur l'alimentation " réunissant les représentants des "puissances possessionnées en Afrique ». Ceux-ci commencent par dresser un état des savoirs sur l'alimentation des populations africaines. Les représentants anglais ont à proposer, pour ce qui est des zones rurales, cinq études menées dans trois villages nigérians et deux villages gambiens ${ }^{61}$. Les représentants belges et portugais ne sont pas en mesure d'apporter la moindre donnée. Les représentants français, enfin, concèdent, pour l'AEF, de faibles connaissances essentiellement fondées sur l'aspect visuel des populations et la production agricole ${ }^{62}$, mais se prévalent, pour l'AOF, d'un savoir nutritionnel unique en Afrique grâce aux investigations menées par la Mission anthropologique ${ }^{63}$.

Si l'on considère ensuite les orientations de recherches de la Mission anthropologique, celles-ci apparaissent traversées par le paradigme de la race: jusqu'à la fin des années 1940, les membres de la Mission posent clairement l'hypothèse d'un déterminisme racial des besoins et des comportements alimentaires. Il faut replacer cette posture dans un plus large contexte: l'idée d'une unicité physiologique des individus, en matière de besoins alimentaires, est alors mal établie. Les premières définitions à l'échelle internationale d'une ration minima standard datent de 1935, avec les normes dites « de Londres » définies par le Comité d’hygiène de la Société des Nations. Dans les faits, elles ne sont utilisées jusqu'à la fin des

${ }^{59}$ Archives de l'Office du Niger, S0166, Organisme d'enquête pour l'étude anthropologique des populations indigènes de l'AOF, «Le bilan de la mission anthropologique de l'AOF (janvier 1946août 1948) », 1948.

${ }^{60}$ PALES, 1949.

${ }^{61}$ D'autres études sont citées par les représentants britanniques mais elles portent toutes sur des collectivités d'écoliers, de militaires ou de salariés urbains. Sur les recherches nutritionnelles menées dans l'Empire britannique dans l'entre-deux-guerres, $c f$. WORBOYs, 1988.

${ }^{62}$ Une thèse novatrice - et citée dans la bibliographie du rapport officiel de la conférence - a pourtant été publiée en 1941 par un pharmacien des troupes coloniales, Paul CRÉAC’H (1993), sur l’alimentation au Tchad.

63 Archives de l’Organisation ouest-africaine de la santé, Fonds Muraz, 2552, Commission de coopération technique au sud du Sahara, « Rapport sur la conférence interafricaine sur l'alimentation et la nutrition, Dschang, Cameroun, 3-9 octobre 1949 », 1950. 
années 1930 que dans des études consacrées à des "populations blanches » ${ }^{64}$. Pour reprendre les termes d'un rapport du Bureau international du Travail sur les standards de vie écrit en 1938, « on ne sait dans quelle mesure les "standards de Londres", qui ont été établis pour l'Europe et l'Amérique du Nord, valent également (ailleurs) : on a constaté que les besoins physiologiques varient avec le climat, le volume de l'organisme humain et l'activité de l'individu (...) et il est possible (qu'ils) diffèrent selon la race » ${ }^{65}$. Dans l'immédiat après-guerre, l'entreprise de standardisation des besoins alimentaires à l'échelle internationale reprend sous l'égide de l'Organisation des Nations unies pour l'Alimentation et l'Agriculture sans que cette entreprise fasse complètement consensus ${ }^{66}$.

Le maniement du paradigme racial par les membres de la Mission anthropologique reflète cependant bien plus que les incertitudes scientifiques du moment vis-à-vis de la notion de "race » et de sa pertinence dans le domaine de l'alimentation : il s'inscrit dans une vieille anthropologie physique qui, largement marginalisée en France métropolitaine, retrouve une nouvelle vitalité dans les colonies ${ }^{67}$. En 1946, Léon Pales met ainsi en place un programme d'étude anthropométrique devant porter sur 139 ethnies de l'AOF ${ }^{68}$. Ce programme consiste à collecter un nombre précis de données biométriques relatives à la stature, aux dimensions de la tête et à celles du nez afin de mettre à jour la «carte ethnique de l'AOF ». Pour reprendre les termes de Léon Pales, ce travail anthropométrique doit permettre de distinguer les « sujets non nègres (...), leurs congénères nigritisés et les Noirs indiscutables » en se fondant sur leurs caractères anatomiques ${ }^{69}$. Cette perspective substantialiste et positiviste place l'anthropologue dans une position surplombante par rapport à ses objets : il lui appartient de rétablir une taxinomie rigoureuse et, au besoin, de redresser les fausses catégories suscitées par les représentations que les populations peuvent avoir d'elles-mêmes ${ }^{70}$.

L'intérêt de cette réactualisation de la carte ethnique de l'AOF n'est pas que spéculatif dans l'optique de Léon Pales. L'enjeu réside dans le croisement de ce travail anthropologique avec des objets d'études pour lesquels la race peut avoir un

${ }^{64}$ Bibliothèque nationale de France, Comité mixte de l'alimentation de la Société des Nations, « Étude sur les politiques nationales de l'alimentation », Genève, 1938.

65 Bibliothèque nationale de France, Bureau international du Travail, «Le standard de vie des travailleurs », Études et documents, série B, n 30, Genève, 1938.

${ }^{66}$ Lors de la première session de l'Organisation des Nations unies pour l'Alimentation et l'Agriculture à Québec, en octobre-novembre 1945, la délégation française défend l’idée de «conditions particulières propres aux pays tropicaux qui, en présentant des réactions physiologiques variant avec les diverses races et les divers milieux, imposent l'obligation de déterminer des normes spécifiques à chaque région pour la défense des rations optima » (Centre des Archives d'outre-mer, 1affpol356, ministère de la France d'outremer, direction du Plan, « Rapport sur la conférence des Nations unies pour l'alimentation et l'agriculture à Québec », 1945)

${ }^{67}$ SUREMAIN, 1999, 39-43.

68 Archives de l'Office du Niger, S0166, Organisme d'enquête pour l'étude anthropologique des populations indigènes de l'AOF, «Le bilan de la mission anthropologique de l'AOF (janvier 1946août 1948) », 1948.

69 « La hauteur du nez », par exemple, est dotée d'une « signification raciale qui isole les Noirs des Méditerranéens et des Ethiopiens » (PALES, 1952b, 54).

${ }^{70}$ Léon Pales recommande ainsi aux enquêteurs de la Mission anthropologique d'avoir la « précaution de ne pas ranger les populations sous les titres qu'ils se donnent ». Au Niger, par exemple, les populations de Tahoua se disent arabes «mais telle n'est pas leur origine », de même que les Bellahs se disent touaregs « alors que, racialement parlant, c’est des Noirs » (PALES, 1952a, 4-5). 
rôle déterminant et, en particulier, avec l'alimentation. Le chef de la Mission anthropologique trouve ainsi «paradoxal (...) que les individus n'aient pas davantage l'aspect de carencés ou de déficients alimentaires sévères » au regard du niveau des rations caloriques calculées. Dès lors, indique-t-il, «il faut bien convenir, ou que l'organisme des Noirs ne fonctionne pas comme le nôtre - et c'est bien possible -, ou bien que l'hypothèse d'apports inconnus extra-familiaux est justifiée - et c'est certain " ${ }^{71}$. Cette hypothèse raciologique est également déclinée dans le domaine de la santé et de la psychologie. Des maladies sont ainsi étudiées à l'aune d'un gradient de «nigritisation» présupposant une prévalence d'autant plus élevée que les populations sont " nigritisées ${ }^{72}$. Des tests d'intelligence, enfin, sont organisés pour mettre en cause l'idée selon laquelle «la capacité intellectuelle est au prorata de l'admission de "sang blanc" voire de couleur plus ou moins foncée de la peau » ${ }^{73}$.

Si l'on considère enfin les résultats de la Mission anthropologique, l'hypothèse du déterminisme racial apparaît globalement écartée, au moins pour ce qui est des besoins alimentaires. Elle est testée pour un certain nombre d'éléments nutritionnels parmi lesquels le glucose, les sels minéraux ou les vitamines. Pour le glucose, par exemple, Léon Pales part du constat d'une hypoglycémie courante chez les populations de l'AOF au regard des normes européennes, avant de se demander si cela ne révèlerait pas une caractéristique naturelle chez «les Noirs » plutôt qu'une carence alimentaire. Suite à ses recherches bio-chimiques, il convient que les "déficiences que l'on observe sur les Africains relèvent d'une alimentation insuffisante » et, en cela, peuvent être qualifiées de " non raciales » ${ }^{74}$. Globalement, l'idée d'une universalité des besoins alimentaires et de l'unicité, en la matière, de la physiologie humaine s'impose dans les conclusions de l'ensemble des travaux de la Mission anthropologique à la fin de la décennie.

Les résultats relatifs à la ration calorique paraissent, quant à eux, plus difficiles à analyser du fait de leur extrême amplitude. La limite inférieure, trouvée dans une famille rurale soudanaise, est de 208 calories par jour ${ }^{75}$. La limite supérieure, trouvée dans une famille de commerçants dakarois, s'élève à 7278 calories par jour soit, pour reprendre les termes de Léon Pales, une «ration à laquelle un organisme humain ne peut résister ${ }^{76}$. Les membres de la Mission anthropologique ne sont pas dupes de la fiabilité de résultats qui doivent beaucoup à l'interaction entre enquêteurs et observés. Ainsi ne cessent-ils de réinterpréter ces résultats en fonction de la manipulation ou des évitements dont ils se disent l'objet. Quand la ration est trop basse, Léon Pales rappelle ainsi qu'elle ne concerne que les « apports visibles » et que les populations

\footnotetext{
${ }^{71}$ Fonds de l'Institut de Médecine tropicale du service de Santé des armées, 101, Organisme de Recherches sur l'Alimentation et la Nutrition africaines, «L'alimentation en AOF. Milieux, enquêtes, techniques, rations ", 1954.

${ }^{72}$ C'est en particulier le cas de la drépanocytose (LinHARD, PALES, 1952).

73 Archives de l'Office du Niger, B0184, Organisme d'enquête pour l'étude anthropologique des populations indigènes de l'AOF, « Rapport n² 2, Soudan occidental et Sénégal », 1946.

${ }^{74}$ Archives de l'Office du Niger, S0166, Organisme d'enquête pour l'étude anthropologique des populations indigènes de l'AOF, «Le bilan de la mission anthropologique de l'AOF (janvier 1946août 1948) », 1948.

${ }^{75}$ Ibid.

${ }^{76}$ Fonds de l'Institut de Médecine tropicale du service de Santé des armées, 101, Organisme de Recherches sur l'Alimentation et la Nutrition africaines, "L'alimentation en AOF. Milieux, enquêtes, techniques, rations », 1954.
} 
observées se sont refusées à mentionner les apports supplémentaires consommés en dehors des repas familiaux ${ }^{77}$. Quand la ration est trop haute, il explique au contraire que les populations ont voulu extérioriser leurs richesses face à des enquêteurs métropolitains ${ }^{78}$.

Cette entreprise de quantification amène, malgré la disparité des données collectées, à dégager quelques grandes tendances. La première réside dans l'importance de la sous-alimentation dans les zones rurales en période de soudure. Cette sous-alimentation saisonnière, si elle est connue des administrateurs, n'a jamais été érigée en objet de mesure. Les chiffres établis, aussi peu fiable soient-ils au regard de leur modalité d'élaboration, ont pour fonction d'alerter les autorités coloniales de l'ampleur du phénomène. La seconde tendance réside dans l'ampleur des disparités nutritionnelles entre les individus. À Dakar, d'importantes différences de niveau d'alimentation sont constatées entre les "personnes dont la situation sociale (est) médiocre » et les " personnes aux revenus à peu près assurés ». Il en va de même dans certains villages, même si «l'incidence sociale du pouvoir d'achat » apparaît moins primordiale ${ }^{79}$. Là encore, aussi peu fiables soient les chiffres, ceux-ci dégagent de fait l'idée, alors largement mésestimée, d’un inégal accès aux ressources alimentaires dans une même collectivité.

Tout en étant portée par une volonté nouvelle de prendre mesure - au sens propre - du problème alimentaire en AOF à l'aune de critères internationaux, la Mission anthropologique apparaît empreinte, tant du point de vue des méthodes que des présupposés de départ, d'une anthropologie physique qui fait de la notion de race l'une des clés fondamentales d'appréhension et de classification des populations. Le savoir nutritionnel produit dans la seconde partie des années 1940 apparaît en cela tiraillé entre une dynamique d'internationalisation impulsée par les organisations onusiennes et un différentialisme colonial toujours marqué.

\section{Les mutations de la recherche nutritionnelle dans les années 1950}

Ce tiraillement s'atténue dans les années 1950 : les investigations nutritionnelles menées en AOF au cours de la décennie se focalisent plus nettement sur l'alimentation des populations au détriment des préoccupations anthropologiques de l'immédiat après-guerre. Cette évolution se traduit par un changement de nom, la Mission anthropologique devenant, en 1953, l'Organisme de Recherches sur l'Alimentation et la Nutrition africaines.

C'est en août 1953 que Bernard Cornut-Gentille, gouverneur général de l'AOF, crée l'Organisme de Recherches sur l'Alimentation et la Nutrition africaines. Les principaux objectifs alloués au nouvel organisme sont de poursuivre l'étude de la sous-alimentation et de la malnutrition en AOF, d'en chercher les causes économiques et sociales et de déterminer des denrées alimentaires à même de pallier

\footnotetext{
${ }^{77}$ Archives de l'Office du Niger, S0166, Organisme d'enquête pour l'étude anthropologique des populations indigènes de l'AOF, «Le bilan de la mission anthropologique de l'AOF (janvier 1946août 1948) ", 1948.

${ }^{78}$ Fonds de l'Institut de Médecine tropicale du service de Santé des armées, 101, Organisme de Recherches sur l'Alimentation et la Nutrition africaines, "L'alimentation en AOF. Milieux, enquêtes, techniques, rations », 1954.

${ }^{79}$ Ibid.
} 
les déficiences constatées. Selon les termes de l'arrêté constitutif, les enquêtes alimentaires seront «éventuellement complétées par des enquêtes ethnologiques et sociologiques (...) destinées à mettre en évidence les causes profondes des déficiences alimentaires ${ }^{80}$. Les références à l'anthropologie physique ont disparu. Tout en prenant la suite de la Mission anthropologique, l'Organisme de Recherches sur l'Alimentation et la Nutrition africaines s'inscrit en cela dans une perspective de recherche différente.

Cette différence s'observe dans l'évolution du profil des chercheurs. Les membres titulaires de l'Organisme de Recherches sur l'Alimentation et la Nutrition africaines sont un peu plus nombreux que ne l'étaient ceux de la Mission anthropologique à sa création puisqu'ils sont au départ 21 médecins, nutritionnistes et chimistes. Si la majorité des médecins reste issue des troupes coloniales, chimistes et nutritionnistes sont extérieurs. Au cours de la décennie, les chercheurs membres d'autres organismes scientifiques, ceux de l'Institut français d'Afrique noire, de l'Office de la Recherche scientifique et technique outre-mer et de l'Institut national de la Statistique et des Études économiques, prennent en outre une part prépondérante dans les travaux de l'Organisme de Recherches sur l'Alimentation et la Nutrition africaines ${ }^{81}$. Celui-ci a beau rester dirigé par un médecin des troupes, le médecin-colonel André Raoult qui était déjà à la tête de la Mission anthropologique, il apparaît moins comme le produit des services de Santé de l'administration coloniale ${ }^{82}$.

L'une des principales activités du nouvel organisme consiste, dans les années 1950, à calculer la composition de la ration moyenne de différentes populations de l'AOF. La question d'un déterminisme racial des besoins alimentaires n'apparaît plus dans les hypothèses de départ, les normes nutritionnelles de références étant clairement posées comme universelles. Cette évolution s'inscrit dans un contexte international d'affirmation de l'universalité des besoins alimentaires. En 1949, un «Comité des besoins en calories » créé au sein de l’Organisation des Nations unies pour l'Alimentation et l'Agriculture avait déjà redéfini les minima standards proposés avant la guerre par le Comité d'hygiène de la Société des Nations. Les différences de besoins considérés par ce comité tenaient aux seuls paramètres du climat, de l'âge et de l'activité physique ${ }^{83}$. En 1957, les membres du deuxième Comité des besoins en calories affirment plus explicitement que «la population du monde se compose de nombreuses races, mais, pour autant qu'on le sache actuellement, des individus de même poids, vivant dans le même milieu et ayant le même mode de vie ont les mêmes

\footnotetext{
${ }^{80}$ Fonds de l'Institut de Médecine tropicale du service de Santé des armées, 340, Gouvernement général de l’AOF, arrêté du 7 août 1953 portant création de l’Organisme de Recherches sur l'Alimentation et la Nutrition africaines.

${ }^{81}$ L’Institut français de l'Afrique noire, créé par un arrêté du 19 août 1936, est la principale institution scientifique en AOF avant la création, en 1943, de l’Office de la Recherche scientifique coloniale qui devient l'Office de Recherche scientifique d'outre-mer en 1944 et l'Office de la Recherche scientifique et technique outre-mer en 1953.

${ }^{82}$ Fonds de l'Institut de Médecine tropicale du service de Santé des armées, 84, Organisme de Recherches sur l'Alimentation et la Nutrition africaines, rapport annuel, 1953. Le docteur Raoult avait succédé en 1951 au docteur Bergougniou, lui-même successeur de Léon Pales en 1949.

83 Bibliothèque nationale de France, Organisation des Nations unies pour l'Alimentation et l’Agriculture, « Rapport du comité des besoins en calories », 1950.
} 
besoins en calories, indépendamment de leur origine ethnique ${ }^{84}$. Si la pertinence de la notion de race reste affirmée, son incidence sur les besoins alimentaires est rejetée.

Les investigations menées par l'Organisme de Recherches sur l'Alimentation et la Nutrition africaines sur la ration calorique ne touchent que la moitié des colonies de l'AOF pendant la décennie. En Haute-Volta, une série d'enquêtes sur les repas familiaux est effectuée dans la partie nord-est du territoire de 1950 à 1952, ainsi que trois études monographiques de villages dans le cercle de Bobo-Dioulasso de 1953 à $1955^{85}$. En Côte d'Ivoire, des monographies de villages sont également faites en 1955-1956 dans la circonscription de Bongouanou ${ }^{86}$. Au Soudan et au Sénégal, enfin, des recherches nutritionnelles sont menées à la fin de la décennie dans le cadre de plus vastes programmes de recherches, la Mission socio-économique du Soudan et la Mission socio-économique du fleuve Sénégal, respectivement organisés dans les cercles de Macina et de Mopti entre 1956 et 1958 et dans le cercle de Saint-Louis en $1957-1958{ }^{87}$.

Le matériau d'enquêtes servant de base à ces recherches reste très limité au début de la décennie. L'enquête réalisée entre 1950 et 1952 sur l'alimentation en HauteVolta est en cela assez significative - et d'autant plus qu'elle continue de faire référence, en matière de connaissances nutritionnelles sur la Haute-Volta jusqu'au milieu des années $1970^{88}$. Dirigée par André Serre, agent technique au service de Santé de la Haute-Volta, elle est menée dans les cercles de Ouahigouya, de Koudougou, de Tougan et de Kaya. Dans chacun de ces cercles, il s'agit de choisir des familles appartenant aux principales ethnies, d'assister au repas et de peser les aliments consommés pendant une semaine et, cela, au moins deux fois dans l'année afin de distinguer les rations dites d'abondance et les rations de soudure. Les rapports finaux, rédigés en 1952, portent sur "l'alimentation des Mossis », " des Samogos », "des Peuls», "des Gourounsis » et "des Gourmantchés» ${ }^{89}$. Chacune de ces catégories repose sur l'observation d'un maximum de 25 familles dont la représentativité - tout comme la catégorisation ethnique - n’est pas discutée.

Les enquêtes réalisées à la fin de la décennie prétendent marquer le franchissement d'une étape méthodologique dans la construction du savoir sur

84 Bibliothèque nationale de France, Organisation des Nations unies pour l'Alimentation et l’Agriculture, « Rapport du deuxième comité des besoins en calories », 1957.

${ }^{85}$ Fonds du Centre Population et Développement, Organisme de Recherches sur l'Alimentation et la Nutrition africaines, " Aspects alimentaires et nutritionnels de la Haute-Volta Est », 1952 ; Archives de l'Organisation ouest-africaine de la santé, fonds Muraz, 2154/3850, Organisme de Recherches sur l’Alimentation et la Nutrition africaines, « Études monographiques de village », 1955.

${ }^{86}$ Centre des Archives d'outre-mer, 2fides766, Organisme de Recherches sur l'Alimentation et la Nutrition africaines, « Enquête nutrition-niveau de vie, subdivision de Bongouanou, 1955-1956 », 1958.

${ }^{87}$ Archives de l’Office du Niger, S0036, Mission socio-économique du Soudan, « L’alimentation des populations rurales du delta vif du Niger et de l’Office du Niger, 1956-1958 », 1961.

${ }^{88}$ Au moment de la crise alimentaire du début des années 1970, la principale référence invoquée dans les rapports internationaux sur la situation nutritionnelle en Haute-Volta avant la crise est une enquête sur les budgets et la consommation publiée en 1966 par l'Institut national de la Statistique et des Études économiques dont une partie des données reprend les résultats de l'étude réalisée entre 1950 et 1952 sous la direction d’André Serre (Fonds du Centre Population et Développement, Direction de la Statistique et de la Mécanographie de la République de Haute-Volta, «Enquête budget-consommation, 1963-1964 », juin 1966).

${ }^{89}$ Fonds du Centre Population et Développement, Organisme de Recherches sur l'Alimentation et la Nutrition africaines, « Aspects alimentaires et nutritionnels de la Haute-Volta Est », 1952. 
l'alimentation en AOF. Les recherches menées entre 1956 et 1958 au Soudan et au Sénégal sont ainsi présentées par leurs auteurs comme les «seules enquêtes alimentaires extensives sur un échantillon probabiliste réalisées dans la zone sahélosoudanienne de l'Ouest africain ${ }^{90}$. Elles sont dirigées, les premières par Pierre Cantrelle, démographe à l'Institut français d'Afrique noire, et les secondes par JeanLouis Boutillier, ethnologue à l'Office de la Recherche scientifique et technique outre-mer. Dans chacun des deux cas, les échantillons ont été établis en fonction de critères variés incluant l'appartenance ethnique mais aussi un certain nombre d'attributs sociaux tels que le profil socioprofessionnel et le niveau d'équipement agricole.

Même si les résultats obtenus restent assez hétérogènes, des tendances générales se dessinent. Les rations moyennes, d'abord, apparaissent sur l'année globalement suffisantes du point de vue de leur valeur calorique mais déficientes du point de vue de leur composition nutritionnelle. Les carences protidiques sont ainsi particulièrement fréquentes dans les villages voltaïques et ivoiriens soumis à l'étude tandis que les avitaminoses sont généralisées dans la zone sahélienne. L'importance des variations saisonnières de la ration, ensuite, est à nouveau soulignée. Le cas de la zone de l'Office du Niger, étudiée dans le cadre de la Mission socio-économique du Soudan, apparaît particulièrement significatif dans la mesure où il s'agit d'une « zone céréalière traversée par un fleuve poissonneux et fréquentée par des éleveurs en bétail » et, qui plus est, supposée bénéficier d'un encadrement hydro-agricole unique en AOF. Or, alors que la ration moyenne s'élève à environ 2300 calories par adulte à l'année, elle s'abaisse à 1800 calories pendant les deux mois de soudure ${ }^{91}$. Si ces chiffres sont très loin d'atteindre les minima calculés dix ans plus tôt par les membres de la Mission anthropologique en zone rurale soudanaise, ils n'en font pas moins apparaître un important problème de sous-alimentation saisonnière puisque celle-ci se manifeste dans une zone a priori moins menacée que la moyenne par la faim.

Un second enseignement tient à l'incidence du profil socio-économique des individus sur leur consommation alimentaire. Cette incidence est d'abord mise en valeur dans l'enquête menée en 1955 en Côte d'Ivoire dans la circonscription de Bongouanou, sous la direction de Jean-Louis Boutillier. L'étude des budgets familiaux fait apparaître que la consommation en protéines animales est directement connectée au niveau de ressources des familles: un niveau supérieur se traduit systématiquement par une plus grande consommation de viande ${ }^{92}$. L'incidence des disparités socio-économiques sur la consommation alimentaire est plus nettement mise en valeur dans l'enquête réalisée entre 1956 et 1958 au Soudan. Une première distinction est faite, dans la zone du delta vif du fleuve Niger, entre les régimes alimentaires des cultivateurs, des pêcheurs et des artisans. Les conclusions apparaissent cependant peu probantes aux dires mêmes des enquêteurs, vu le très

\footnotetext{
${ }^{90}$ Archives de l’Office du Niger, S0036, Mission socio-économique du Soudan, «L'alimentation des populations rurales du delta vif du Niger et de l’Office du Niger, 1956-1958 », 1961.

${ }^{91}$ Ibid.

${ }^{92}$ Centre des Archives d'outre-mer, 2fides766, Organisme de Recherches sur l'Alimentation et la Nutrition africaines, «Enquête nutrition-niveau de vie, subdivision de Bongouanou, 1955-1956 », rapport $n^{\circ} 1$, « Budgets », septembre 1955. L'enquête sur les budgets familiaux dans la subdivision de Bongouanou repose sur une observation d'une à deux semaines de 2450 personnes, chacune de leurs transactions, financière ou non, étant enregistrée.
} 
faible nombre d'enquêtes réalisées pour les deux dernières catégories ${ }^{93}$. Une seconde distinction plus significative est faite, toujours dans la zone du delta vif mais à partir d'un plus large échantillonnage, entre cultivateurs avec charrue et cultivateurs sans charrue. Si la ration annuelle apparaît, en moyenne, largement excédentaire chez les premiers, elle est à peine suffisante chez les seconds ${ }^{94}$. Le niveau d'alimentation des familles apparaît ainsi tributaire de l'outillage dont elles disposent pour cultiver la terre.

À la fin des années 1950, l'AOF apparaît plus nettement associée à la sousalimentation qu'elle ne l'était vingt ans auparavant. Cette évolution ne découle pas d'une brusque découverte de la faim par les administrateurs coloniaux, comme s'ils n'avaient nulle conscience du phénomène dans l'entre-deux-guerres. Elle résulte du développement dans les colonies ouest-africaines d'une recherche nutritionnelle qui, jusqu'alors, ne les avait presque pas touchées. Ce qui est nouveau, dans l'aprèsguerre, ce n'est pas tant la prise de conscience de la faim par les administrateurs que le souci de la mesurer à l'aune d'indicateurs standardisés.

Dans la première partie des années 1970, la sécheresse qui affecte l’Afrique sahélienne donne lieu à de nombreuses études destinées à en évaluer les effets sur les niveaux d'alimentation des populations ${ }^{95}$. Pareille évaluation suppose de comparer ce qui est posé comme un état de crise à ce qui aurait dû être la norme dans des conditions pluviométriques moyennes. La sécheresse suscite donc une mobilisation des savoirs existants sur l'alimentation des populations sahéliennes avant la crise. Or, ces savoirs apparaissent extrêmement minces. Les principales enquêtes nutritionnelles disponibles pour des pays tels que le Mali ou la Haute-Volta remontent aux années 1950 : il s'agit de celles réalisées sous l'égide de l'Organisme de Recherches sur l'Alimentation et la Nutrition africaines en fin de période coloniale. La brusque multiplication des investigations nutritionnelles en temps de crise met ainsi en exergue, par contraste, une méconnaissance persistante sur l'alimentation ordinaire des populations sahéliennes.

Le contraste apparaît particulièrement saisissant en matière d'étude clinique de la faim et de la malnutrition. Plusieurs enquêtes sont faites à partir de 1973 pour évaluer la fréquence des carences nutritionnelles en Afrique sahélienne, à partir d'indicateurs tels que le périmètre thoracique, la circonférence des bras ou le rapport poids-taille. Les données enregistrées sont ensuite rapportées à des normes standards reconnues par les organisations internationales afin d'évaluer la proportion de sous-alimentés et de malnutris parmi les populations étudiées ${ }^{96}$. Ces enquêtes mettent en valeur une forte prévalence de la sous-nutrition sur les territoires affectés par la sécheresse. Mais elles ne permettent pas d'évaluer à quel point cette prévalence est inhabituelle, pour la simple raison qu'il n'existe pas d'enquêtes cliniques antérieures qui leur soient

\footnotetext{
9312 enquêtes sont faites dans des familles de pêcheurs et 16 dans des familles d'artisans. Cette comparaison fait apparaître un meilleur apport en protéines animales chez les pêcheurs que dans les autres groupes socioprofessionnels.

${ }^{94}$ Archives de l’Office du Niger, S0036, Mission socio-économique du Soudan, « L’alimentation des populations rurales du delta vif du Niger et de l’Office du Niger, 1956-1958 », 1961.

${ }^{95}$ Jean Roch cite l’essentiel de ces études dans sa « Bibliographie sélective sur les famines récentes au Sahel » (RoCH, 1975).

${ }^{96}$ Plusieurs standards définis après la guerre et reconnus par les organisations internationales sont repris dans ces investigations, en particulier la courbe poids-taille Stuart-Meredith ou les critères biométriques de Jeliffe.
} 
comparables ${ }^{97}$. Si les enquêteurs s'accordent, au moins à partir de l'année 1973, pour reconnaître la gravité de la situation alimentaire dans les pays sahéliens, ils apparaissent ainsi incapables de préciser ce que cette situation doit à la sécheresse.

Ceci amène à relativiser l'évolution générale dont il vient d'être question ici. Certes, des années 1920 aux années 1950, une nouvelle approche de la question alimentaire se dessine au sein de l'AOF. Au savoir productif, quasiment exclusif jusque dans les années 1930, vient se mêler un savoir nutritionnel qui, encore empreint du paradigme racial dans la seconde partie des années 1940, se fonde sur des indicateurs posés comme universels au cours des années 1950. Ce savoir n’en demeure pas moins limité au moment des indépendances. Finalement, les choses ne changent pas tant que ça, sinon au niveau des cadres cognitifs à partir desquels est pensée la question alimentaire en Afrique de l'Ouest : les Africains et les Européens se mettent à avoir les mêmes besoins alimentaires après la Seconde Guerre mondiale mais la manière dont ces besoins sont effectivement assouvis en période ordinaire demeure mal connue.

Vincent BONNECASE Centre d'Étude d'Afrique Noire, Bordeaux, France vincentbonnecase@yahoo.fr

\section{Bibliographie}

Ageron Ch.R., Coquery-Vidrovitch C., Meynier G., Thobie J., 1990, Histoire de la France coloniale, Paris, Armand Colin.

AlmEIDA TOPOR H. d', 1986, Cultures vivrières et faim en AOF pendant la période coloniale, in BRunchaus E., HARDing L., (eds.), Hundert Jahre Einmischung in Afrika, 1884-1984, Hamburg, Helmut Buske Verlag, 309-319.

AlPHA GADO B., 1988, Crises alimentaires et stratégies de subsistance en Afrique sahélienne (Mali, Burkina Faso, Niger) aux XIX ${ }^{e}$ et $X x^{e}$ siècles, Thèse de $3^{\mathrm{e}}$ cycle, Paris, Université de Paris VII.

AlPhA GADO B., 1993, Une histoire des famines au Sahel. Étude des grandes crises alimentaires. $X I X^{e}-X X^{e}$ siècles, Paris, l'Harmattan.

AlPHA GADO B., 1996, La situation alimentaire en Haute-Volta dans la première moitié du $\mathrm{Xx}^{\mathrm{e}}$ siècle. Les cas des cercles de Dori et de Ouahigouya, in MAdiÉGA G., NAO O., (dir.), Burkina Faso, cent ans d'histoire, 1895-1995, Actes du $1^{\mathrm{er}}$ colloque international sur l’histoire du Burkina Faso, Paris, Karthala-PUO, 1335-1351.

BeuseKom M. van, 2000, Disjuncture in Theory and Practise : Making Sense of Change in Agriculture Development at the Office du Niger, 1920-1960, The Journal of African History, 41, 1, 79-99.

BonneCase V., 2008, Pauvreté au Sahel. La construction des savoirs sur les niveaux de vie au Burkina Faso, au mali et au Niger (1945-1974), Thèse de doctorat, Paris, Université de Paris I.

BuRNETT J., 1995, Les enquêtes sur l'alimentation et la mesure de la pauvreté (1790-1945), in CARRÉ J., RÉvAUger J.P., (dir.), Écrire la pauvreté. Les enquêtes sociales britanniques aux $X I X^{e}$ et $X X^{e}$ siècles, Paris, l'Harmattan, 145-170.

\footnotetext{
${ }^{97}$ BONNECASE, 2008, 343-348.
} 
CHASTANET M., 1983, Les crises de subsistance dans les villages soninkés du cercle de Bakel de 1858 à 1945, Cahier d'Études Africaines, XXIII, 89-90, 5-36.

CoHen W., 1972, The Colonial Policy of the Popular Front, French Historical Studies, VII, 3, 368-393.

Conklin A., 1997, A Mission to Civilize. The Republican Idea of Empire in France and West Africa, 1895-1930, Stanford, Stanford University Press.

Coquery-Vidrovitch C., 1999, The Popular Front and the Colonial Question. French West Africa : an Example of Reformist Colonialism, in CHAFER T., SACKUR A., French Colonial Empire and the Popular Front : Hope and Disillusion, Londres, McMillan, 155-169.

CRÉAC'H P., 1999, Se nourrir au Sahel. L'alimentation au Tchad, Paris, l'Harmattan.

Cullather N., 2007, The Foreign Policy of the Calorie, American Historical Review, 112, 2, 337-364.

DakuYo N., Lougué H., 1984, La Haute-Volta, in Groupe de Démographie Africaine, L'évaluation des effectifs de la population des pays africains, Paris, ORSTOM, $2^{\mathrm{e}}$ tome, 169-178.

Destombes J., 2001, Nutrition and Chronic Deprivation in the West African Savanna : NorthEastern Ghana, 1930-2000, Ph.D., London, London School of Economics and Political Science.

DupIN H., 1969, Les enquêtes nutritionnelles, méthodes et interprétation des résultats (à propos des enquêtes faites en Afrique), Paris, CNRS Éditions.

EGG J., (ed.), 1975, Analyse descriptive de la famine de 1931 au Niger et implication méthodologique, Paris, INRA.

Filipovich J., 1985, The Office du Niger under Colonial Rule: its Origine, Evolution and Character (1920-1960), Ph.D., Montréal, McGill University.

FUGLESTAD F., 1974, La grande famine de 1931 dans l'Ouest nigérien : réflexions autour d'une catastrophe naturelle, Revue Française d'Histoire d'Outre-Mer, LXVI, 222, 18-33.

HARDY G., RicheT Ch., (dir.), 1933, L'alimentation indigène dans les colonies françaises, protectorats et territoires sous mandats, Paris, Vigot Frères Éditeurs.

LABOURET H., 1938, Famines et disettes aux colonies, Première conférence internationale pour la protection contre les calamités naturelles, Paris, 13-17 septembre 1937, extraits, Paris, Secrétariat de la Conférence.

LHUISSIER A., 2007, Alimentation populaire et réforme sociale. Les consommations ouvrières dans le second XIX ${ }^{e}$ siècle, Paris, Éditions de la Maison des Sciences de l’Homme.

LinHARD J., PALES L., 1952, La sicklémie en AOF vue de Dakar, L'Anthropologie, 56, 1-2, 53 85.

MASSA G., 2003, Le développement économique, in ClAUZEL J., (dir.), La France d'Outre-mer (1930-1960). Témoignages d'administrateurs et de magistrats, Paris, Karthala, 165-210.

PALES L., 1949, Parallèle anthropométrique succinct (stature) des militaires et des civils, Raciologie Comparative des Populations de l’AOF, I, Paris, Bulletins et Mémoires de la Société d'Anthropologie de Paris.

Pales L., 1952a, Anatomie sommaire des Maures de l'AOF et notamment des Maures du Trarza, Raciologie Comparative des Populations de l'AOF, III, Paris, Bulletins et Mémoires de la Société d'Anthropologie de Paris.

PAles L., 1952b, Parallèle anatomique succinct des Maures de l’AOF, notamment des Maures du Trarza avec des Peuls et des Ouolof, Raciologie Comparative des Populations de l'AOF, IV, Paris, Bulletins et Mémoires de la Société d’Anthropologie de Paris. 
Roch J., 1975, Bibliographie sélective sur les famines récentes et la sécheresse au Sahel, Environnement Africain, 1, 2, 102-124.

Roubaud É., 1926, Rapport sur la Question de l'Insuffisance alimentaire des Indigènes dans les Possessions françaises, présenté sur la proposition du Dr Calmette, in ACADÉMIE DES Sciences Coloniales, Compte-rendus des Séances. Communications, Paris, Société d’Éditions Géographiques, Maritimes et Coloniales, tome IV, 357-376.

SALIFOU A., 1975, Quand l'histoire se répète : la famine de 1931 au Niger, Environnement Africain, I, 2, 25-53.

SiBEUd E., 2002, Une science impériale pour l'Afrique? La construction des savoirs africanistes en France, 1878-1930, Paris, Édition de l'EHESS.

Simmons D.J., 2004, Minimal Frenchmen: Science and Standards of Living, 1840-1960, Ph.D., Chicago, University of Chicago.

SuREMAIN M.A. de, 1999, Cartographie coloniale et encadrement des populations en Afrique, Revue Française d'Histoire d'Outre-Mer, 324-325, 29-64.

Worboys M., 1988, The Discovery of Colonial Malnutrition between the Wars, in ARNOLD D., (ed.), Imperial Medicine and Indigenous Societies : Disease, Medicine and Empire in the Nineteenth and Twentieth Centuries, Manchester, Manchester University Press, 208-225. 